\title{
Kottamia Faint Imaging Spectro-Polarimeter (KFISP): opto-mechanical design, software control and performance analysis
}

\author{
Yosry A. Azzam, et al. [full author details at the end of the article]
}

Received: 10 February 2021 / Accepted: 23 September 2021 / Published online: 13 October 2021

(c) The Author(s), under exclusive licence to Springer Nature B.V. 2021

\begin{abstract}
In this paper we describe the Kottamia Faint Imaging Spectro-Polarimeter (KFISP) that has been recently developed and designed to be mounted at the Cassegrain focus of the $1.88 \mathrm{~m}$ telescope at Kottamia Astronomical Observatory (KAO), Egypt. The optical design of KFISP is developed such that it can be used in various modes of operation. These are: direct imaging, spectroscopic, polarimetric imaging, and spectro-polarimetric. The KFISP is an all-refractive design to meet the polarimetric requirements and includes a focal reducer with a corrector section, collimator section, parallel beam section (containing various imaging components), and camera section. The corrector section gives an unvignetted Field-of-View of $8^{\prime} \times 8^{\prime}$ and the collimator section has a focal length of $305 \mathrm{~mm}$ and matches the focal ratio of the input beam. The parallel beam section is $200 \mathrm{~mm}$ long and near the middle of it there is an image of the telescope pupil. The camera section includes 5 elements and has a focal length of $154.51 \mathrm{~mm}$ which gives an instrument effective final focal ratio of f/6.14 (acting as a telescope focal reducer of 1:2 ratio). The KFISP contains an internal calibration system which hosts the calibration light injection system, an integrating sphere equipped with the required calibration light sources. The opto-mechanical parts of KFISP contain a double-layered carbon fiber strut structure and comprises its subsystems of slit and guider assemblies, filter wheel drawer, grism wheel drawer, polarimetric components cubical box, and CCD camera which is integrated with camera optics. The CCD camera has $2048 \times 2048$ pixels with 13.5-micron square pixel size. The camera is cooled by liquid Nitrogen and is fixed to the KFISP through the integrated camera lens. The KFISP has been fully commissioned, mounted and is being tested in all modes of operation. In this paper we introduce the ambitious scientific goals, the optical setups of KFISP, its opto-mechanical implementation and the performance analysis of the instrument. In addition, we describe the camera system, its performance, and its software control. Finally, we present a sample of the first light observations obtained from the instrument.
\end{abstract}

Keywords Kottamia observatory · Instrumentation · KFISP · Spectrograph · Direct imaging $\cdot$ Spectroscopy $\cdot$ Polarimetry 


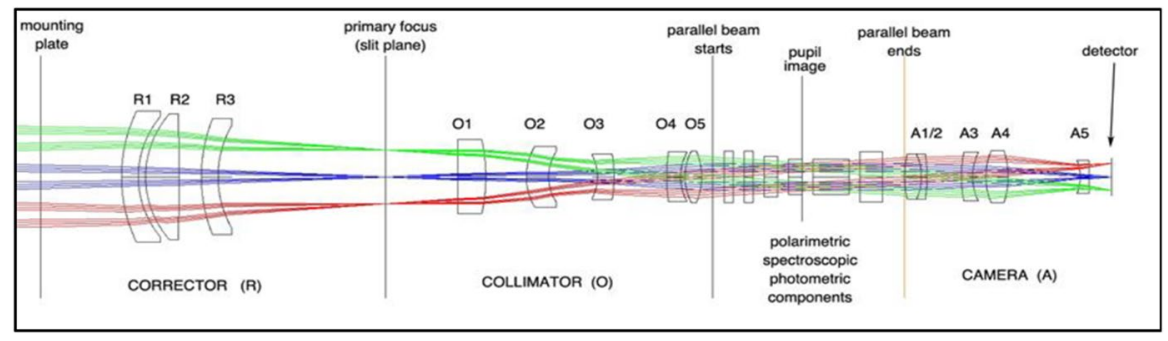

Fig. 1 The optical layout of the KFISP, identifying the components and sub-assemblies

\section{Introduction}

The Kottamia $1.88 \mathrm{~m}$ telescope was erected in 1963 at Kottamia Astronomical Observatory (KAO) which lies about $80 \mathrm{Kms}$. NE of Cairo, $476 \mathrm{~m}$ above sea-level [1]. The latitude of the observatory is $29^{\circ} 55^{\prime} 35.24^{\prime \prime} \mathrm{N}$; the longitude is $31^{\circ} 49^{\prime}$ 45.85" E. The average prevailing seeing conditions at the site is around 2 arcsec [2]. KAO belongs to the National Research Institute of Astronomy and Geophysics; NRIAG (previously, Helwan Observatory). The telescope has a f/4.9 parabolic primary mirror with secondaries to give f/4.9 Newtonian, f/18 Cassegrain and Coudé foci [3]. Telescope optical system was upgraded in 2003 by Zeiss company (FRG) with a new primary mirror, secondary Cassegrain mirror both are made of "Zerodur" glass ceramics, and a new supporting system for the primary mirror [4].

Following the optical system upgrade, a refurbishment process for the whole telescope control system was implemented in 2008. This refurbishment included replacing the existing control cabinet with the up-to-date equipment enabling computer control of all aspects of the dome and telescope [1].

The telescope has been used at Newtonian and Cassegrain stations but the narrow field of view at Cassegrain and the inconvenience of mounting equipment at Newtonian led to the development of the KFISP, a single multi-purpose Cassegrain instrument, incorporating a focal reducer to create an effective f/6 telescope [5].

\section{Optical design of KFISP}

The Kottamia Faint Imaging Spectro-Polarimeter (KFISP) was custom-designed to work on the $1.88 \mathrm{~m}$ Cassegrain telescope of the Kottamia Astronomical Observatory (KAO). It is an all-refractive design to meet the polarimetric requirements, and thus directly mounts on the Cassegrain focal station. The basic concept of this optical layout follows optical versatile instruments such as HOWPol [6] at 1.5-m Kanata Telescope and FOCAS [7] at 8.2-m Subaru Telescope. The optical layout is presented in Fig. 1 in which we can see no folds which is a design requirement of polarimetry and its small instrumental polarization [5]. Some of the quantitative design requirements can be summarized as follows: 
Field of View: $>8$ arcmin $\times 8$ arcmin; pixel scale 0.25 arcsec/pixel; observable wavelength: $350-900 \mathrm{~nm}$; total transmission: (50-70\%) for the wavelength range $350-450 \mathrm{~nm}$ and higher than $70 \%$ for the wavelength range $450-900 \mathrm{~nm}$; radius of $80 \%$ encircled energy: $80 \%$ encircled energy diameter should be $<15 \mu \mathrm{m}$ in the wavelength range 350-900 $\mathrm{nm}$ everywhere within the FoV; image distortion should be $<5 \%$ between the center and at the edge of the FoV; capabilities: direct imaging, spectroscopy, polarimetry imaging; both linear and circular polarisation should be measured by the polarimetric imaging device; length: $<1.4 \mathrm{~m}$; weight: $<350 \mathrm{~kg}$.

\subsection{Corrector and slit plane design}

The corrector acts as a focal reducer, creating a f/12.06 beam with a well-corrected field of view of $8 \times 8$ arc minutes. The resulting focus or slit plane is sufficiently far behind the corrector assembly to accommodate a guider and slit viewing optics. The plate scale at the slit plane is $9.06 \mathrm{arcsec} / \mathrm{mm}$. The corrector section is comprised of 3 optical elements (R1-R3), all of spherical design which are made of Ohara type i-Line good blue transmission glasses (https://www.oharacorp.com/iline. html). Table 1 lists all the lens elements (R1-R3 for the corrector, O1-O5 for the collimator, A1-A5 for the camera), with their diameter, radii of curvatures, central thickness, spacing and glass material. The front element of the corrector (lens R1) has a diameter of $150 \mathrm{~mm}$ which is giving the previously mentioned values of FoV. The guider field is still usable, unvignetted to 12 arcminutes FoV with $30 \%$ transmission to 15 arcminutes FoV diameter as is shown in Fig. 2. The image quality at the primary focus showing the corrector performance is given by the spot diagram of Fig. 3. It is a good match to the expected seeing conditions and telescope performance and will not degrade the images.

\subsection{Collimator design}

Following the slit plane is the collimator section, consisting of 5 lens elements (O1-O5). The lens train has a maximum diameter of $90 \mathrm{~mm}$ and is $200 \mathrm{~mm}$ long. The collimator has a focal length of $305.23 \mathrm{~mm}$ and matches the focal ratio of the input beam. Thus, at $\mathrm{f} / 12.0$ and $\mathrm{f}=305.23 \mathrm{~mm}$ the collimated beam diameter is $28.4 \mathrm{~mm}$ at the pupil image (center of parallel beam section being described in Sect. 2.4 below). It should be noted here that the lenses of the corrector and collimator sections may cause an instrumental polarization for off-axis sources, but it should be stable and can be subtracted by a proper calibration process.

\subsection{Camera section}

The camera section is a 5-element spherical design (A1-A5) with a maximum diameter of $70 \mathrm{~mm}$ and a total length of $175 \mathrm{~mm}$. These elements are of Ohara i-Line glasses, CaF2, one S-LAL12 element at $7 \mathrm{~mm}$ thickness with $92.2 \%$ internal transmission at $350 \mathrm{~nm}$. The effective focal length of the camera section is $154.51 \mathrm{~mm}$, resulting in an effective focal ratio of the instrument to be $f / 6.14$, or reducing the 
Table 1 Lens elements with their diameter, radii of curvatures, central thickness, spacing and glass material used in KFISP

\begin{tabular}{|c|c|c|c|c|c|c|}
\hline Lens & Diameter (mm) & $\mathrm{r} 1$ & r2 & $\mathrm{CT}$ & Spacing & Material \\
\hline $\mathrm{R} 1$ & 150 & 197.10 & 115.65 & 15.08 & & BAL15Y \\
\hline R1-R2 & & & & & 7.77 & \\
\hline $\mathrm{R} 2$ & 145 & 119.18 & -1171.87 & 34.99 & & $\mathrm{CaF} 2$ \\
\hline R2-R3 & & & & & 37.02 & \\
\hline R3 & 130 & 175.30 & 117.42 & 15.10 & & BSL7Y \\
\hline O1 & 90 & Plano & 144.19 & 15.08 & & BSM51Y \\
\hline $\mathrm{O} 1-\mathrm{O} 2$ & & & & & 16.65 & \\
\hline $\mathrm{O} 2$ & 80 & 61.128 & -48.602 & 12.08 & & PBM8Y \\
\hline $\mathrm{O} 2-\mathrm{O} 3$ & & & & & 55.20 & \\
\hline $\mathrm{O} 3$ & 64 & -57.624 & 122.914 & 12.04 & & BAL15Y \\
\hline O3-O4 & & & & & 36.87 & \\
\hline $\mathrm{O} 4$ & 64 & 1275.57 & -56.51 & 11.95 & & BAL35Y \\
\hline O4-O5 & & & & & 4.41 & \\
\hline O5 & 62 & 63.721 & 60.205 & 22.02 & & $\mathrm{CaF} 2$ \\
\hline A1 & 68 & 82.28 & 65.19 & 22.98 & & $\mathrm{CaF} 2$ \\
\hline $\mathrm{A} 1-\mathrm{A} 2$ & & & & & 0.64 & \\
\hline $\mathrm{A} 2$ & 68 & -63.70 & 204.57 & 7.088 & & S-LAL12 \\
\hline $\mathrm{A} 2-\mathrm{A} 3$ & & & & & 22.21 & \\
\hline A3 & 70 & 121.603 & -56.188 & 8.061 & & BSL7Y \\
\hline A3-A4 & & & & & 14.60 & \\
\hline A4 & 70 & 64.791 & 98.498 & 22.957 & & $\mathrm{CaF} 2$ \\
\hline A4-A5 & & & & & 54.15 & \\
\hline A5 & 56 & -40.92 & Plano & 8.04 & & BAL35Y \\
\hline
\end{tabular}

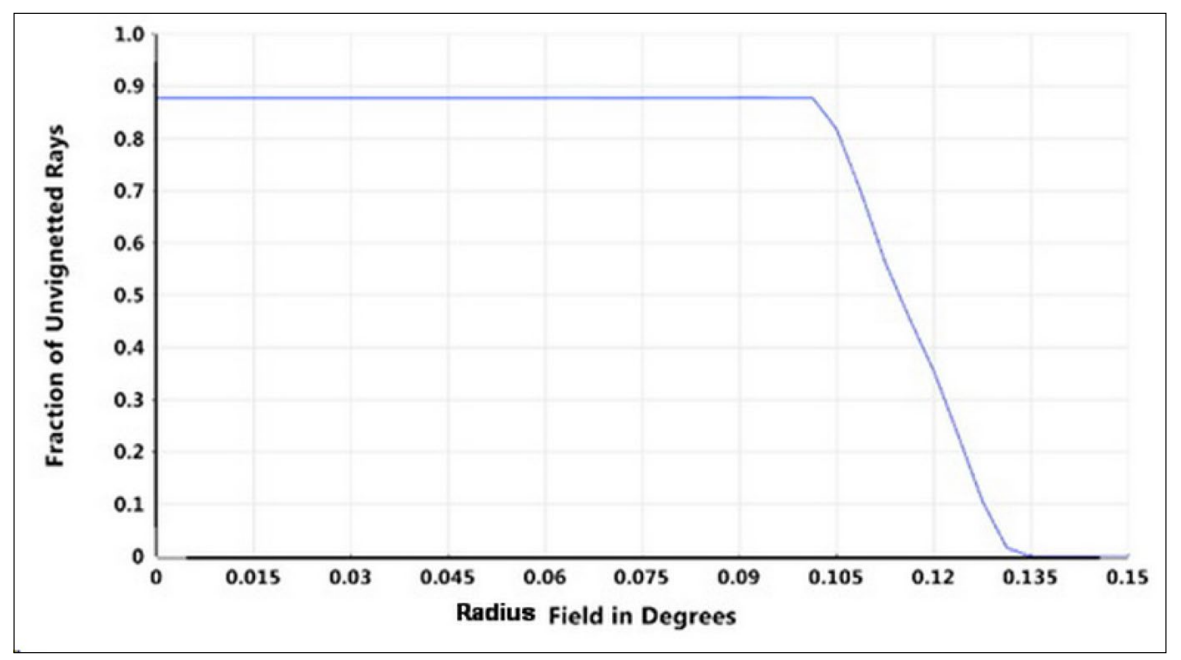

Fig. 2 Guider field fraction of unvignetted rays 


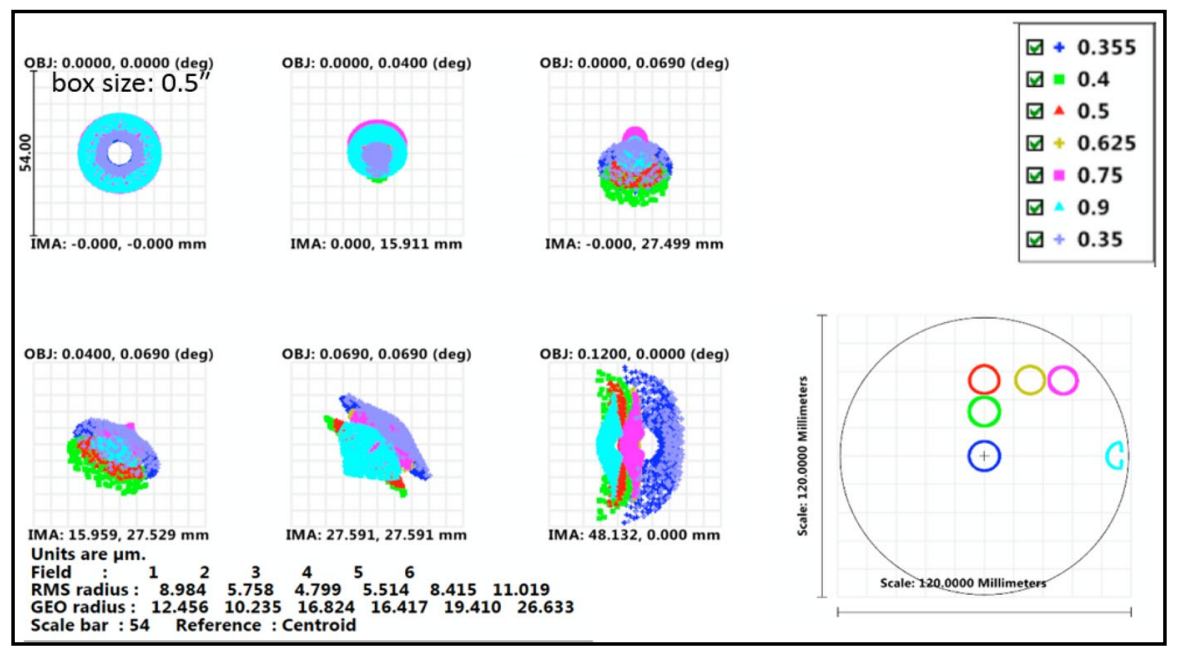

Fig. 3 Spot diagrams for the primary focus field imaging performance

telescope effective focal ratio by a factor of 2 . This is equivalent to a plate scale of $17.78 " / \mathrm{mm}$ or $0.24 " /$ pixel with a standard 13.5 -micron pixel size (E2V detector). With a $2048 \times 2048$ pixel device ( $2 \mathrm{k} \times 2 \mathrm{k} \mathrm{CCD}$ ), the covered field of view is $8.2 \times 8.2$ arcminutes. The optical elements of the camera are integrated to CCD camera as the last element acts as the dewar window.

\subsection{Parallel beam section}

The collimated beam section is $200 \mathrm{~mm}$ long and the pupil image is $28.4 \mathrm{~mm}$ at the center of collimated beam section (at the 2 nd waveplate). Here, all beams (all wavelengths from all field angles/positions) pass through the aperture at the pupil position. The position of the pupil is slightly dependent on what components are inserted into the collimated beam. The nominal position and size of the pupil, along with the order of the components within the parallel beam, was chosen so that the size and thus cost of the most expensive optical elements (waveplates and polarizer) can be minimized. The pupil image is placed over the grating/grism, which in this design is somewhat compromised for optimizing cost.

The collimated beam hosts the following components, as each appears from left to right in the parallel beam section:

A. Filter \#1, $50.8 \mathrm{~mm}$ in diameter (typically photometric filters).

B. Filter \#2, $50.8 \mathrm{~mm}$ in diameter (typically order-sorting filters).

C. $\lambda / 2$ waveplate, $40 \mathrm{~mm}$ in diameter.

D. $\lambda / 4$ waveplate, $40 \mathrm{~mm}$ in diameter.

E. Polarizer (Wollaston prism), $41 \times 41 \mathrm{~mm}$ diameter.

F. Grism, $50 \times 50 \mathrm{~mm}$ cross section and variable central thickness between 10 and $20 \mathrm{~mm}$. 
Table 2 Possible observing modes

\begin{tabular}{|c|l|c|c|c||c|c||c||c|}
\hline mode & \multicolumn{1}{|c|}{ observing mode } & OPT & \multicolumn{4}{|c|}{ components in beam } \\
\cline { 5 - 8 }$\#$ & & {$[\mathbf{y} / \mathbf{n}]$} & A & B & C & D & E & F \\
\hline 1 & White light imaging & $\mathrm{y}$ & & & & & & \\
\hline 2 & Photometric imaging & $\mathrm{y}$ & $\mathrm{X}$ & & & & & \\
\hline 3 & Polarimetric imaging with filters & $\mathrm{y}$ & $\mathrm{X}$ & & $\mathrm{X}$ & $\mathrm{X}$ & $\mathrm{X}$ & \\
\hline 4 & Spectroscopy & $\mathrm{y}$ & & $\mathrm{X}$ & & & & $\mathrm{X}$ \\
\hline 5 & Spectro-polarimetry & $\mathrm{y}$ & & $\mathrm{X}$ & $\mathrm{X}$ & $\mathrm{X}$ & $\mathrm{X}$ & $\mathrm{X}$ \\
\hline
\end{tabular}

back side of

Wollaston

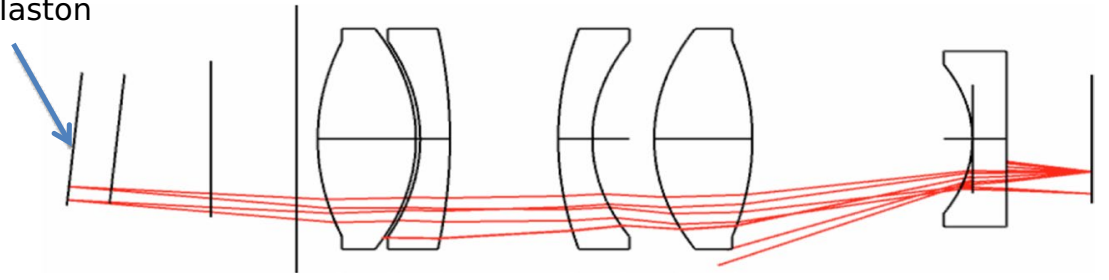

Fig. 4 An example of ghost reflection on CCD

The optical design was optimized in such way that image quality in many of the possible observing modes were evaluated simultaneously (in accordance with the component list above). The operating modes of KFISP are as listed in Table 2.

\subsubsection{Filters and grisms}

KFISP contains two filter wheels with ten positions in each of them to use 18 filters and leaving two positions for white light imaging. Filters are tilted at $5^{\circ}$ to eliminate ghost reflection on the CCD. Figure 4 shows an example case for light reflecting back from CCD, traveling to Wollaston prism, then coming back to detector, but missing the image on this 2nd pass.

The filters we are using in KFISP are three groups of Johnson (U, B, V, R), Stromgren (u,b,v,y), and SDSS ( $\mathrm{u}, \mathrm{g}, \mathrm{r}, \mathrm{i}, \mathrm{z})$ comprising wide-band, narrow-band and SDSS filters. The specifications of those groups are included in Tables 3 and 4.

The grisms are ruled gratings replicated on the back of glass prisms. The entrance face of each prism is AR coated and the prism material is an UV transmissive version of BK7 glass (I-BSL7Y). This way the index of refraction of the epoxy resin, used for the grating replication, will be a close match to the glass ensuring minimal internal reflection losses at the glass-resin boundary.

Keeping in mind the size of the telescope and the seeing conditions ( 2 "), the optical design of the spectroscopic part was made as simple as possible and the set of grisms presented in Table 5 was chosen.

As can be seen from the table, a blue and a red grating were selected for both the low (L) and medium (M) resolutions, and these gratings are used very close to their blaze angle. The central wavelength for the blue spectroscopic modes is $450 \mathrm{~nm}$, and by use of a low-pass filter the blue spectrum will cover the 350-600 nm range in a clean first order spectrum. The second order overlap would be diluting the spectrum 
Table 3 Specifications of Jonson and Stromgren filters used in KFISP

Table 4 Specifications of SDSS filters used in KFISP

\begin{tabular}{llll}
\hline Filter group & Band & Filter & $\begin{array}{l}\text { Central } \\
\text { wavelength } \\
(\mathrm{nm})\end{array}$ \\
\hline Johnson & Wide & U & 363 \\
& & B & 420 \\
& V & 530 \\
Stromgren & Rarrow & u & 597 \\
& & b & 357 \\
& v & 465 \\
& y & 415 \\
& H $\alpha$ & 537 \\
& OIII & 656.3 \\
& & 500.7 \\
\hline
\end{tabular}

\begin{tabular}{llll}
\hline Filter group & Filter & Effective $\lambda(\mathrm{nm})$ & FWHM $(\mathrm{nm})$ \\
\hline \multirow{2}{*}{ SDSS } & u' & 355 & 60 \\
& g' & 468 & 140 \\
& r' & 617 & 140 \\
& i' & 748 & 150 \\
& z' & 893 & 100 \\
\hline
\end{tabular}

starting at $700 \mathrm{~nm}$, but since the grating efficiency would be very low there a cut at $600 \mathrm{~nm}$ was decided. The long wavelength portion of the spectra can be observed in the red settings, using a long-pass filter starting to cut in around $500 \mathrm{~nm}$. Thus, the red spectrum covers the $500-900 \mathrm{~nm}$ range with a $720 \mathrm{~nm}$ central wavelength as a clean 1 st order spectrum with no contamination from the 2 nd order.

\subsubsection{Waveplates}

Commercial off-the-shelf superachromatic retarders from Bernhard Halle Nachfl, GMBH were used (https://www.b-halle.de/products/retarders/superachromatic_retar ders.html). These are Pancharatnam-type super-achromatic waveplates [8]. Both the $\lambda / 2$ and $\lambda / 4$ components are $16 \mathrm{~mm}$ thick in the shape of a $42 \mathrm{~mm}$ across flats octagon. They are made of $\mathrm{MgF}_{2}$ and quartz crystal cemented between cover plates made of fused silica, with a beam deviation of less than 1 arc minute and have multilayer antireflection coatings optimized for 350-900 $\mathrm{nm}$. This involves polishing of the crystalline quartz and magnesium-fluoride plates (6 in total, 12 surfaces) to a very precise thickness of a few hundred microns each with a high degree of parallelism [9]. Figure 5 shows the design of both $1 / 2$ and $1 / 4$ waveplates that represent curves for retardation, axis angle and polarization loss as a function of wavelength. The solid red curve is a custom design, optimized for the $350-900 \mathrm{~nm}$ wavelength range, 


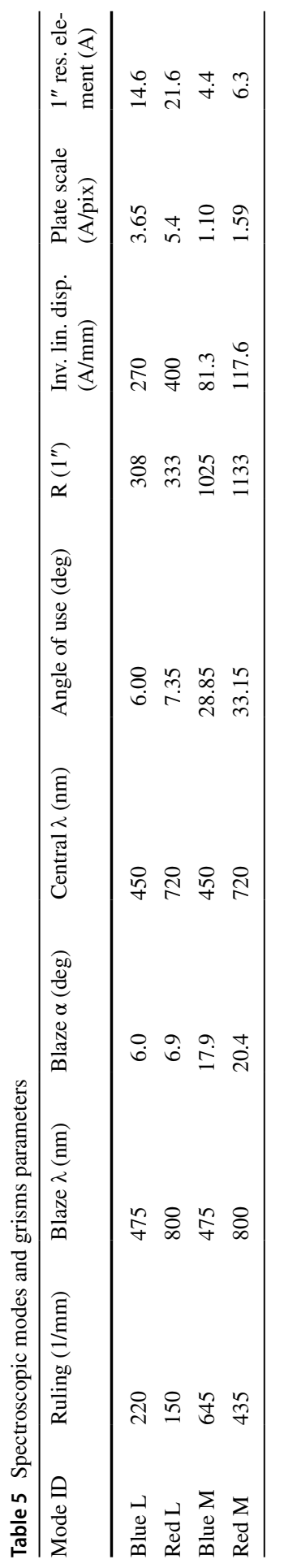




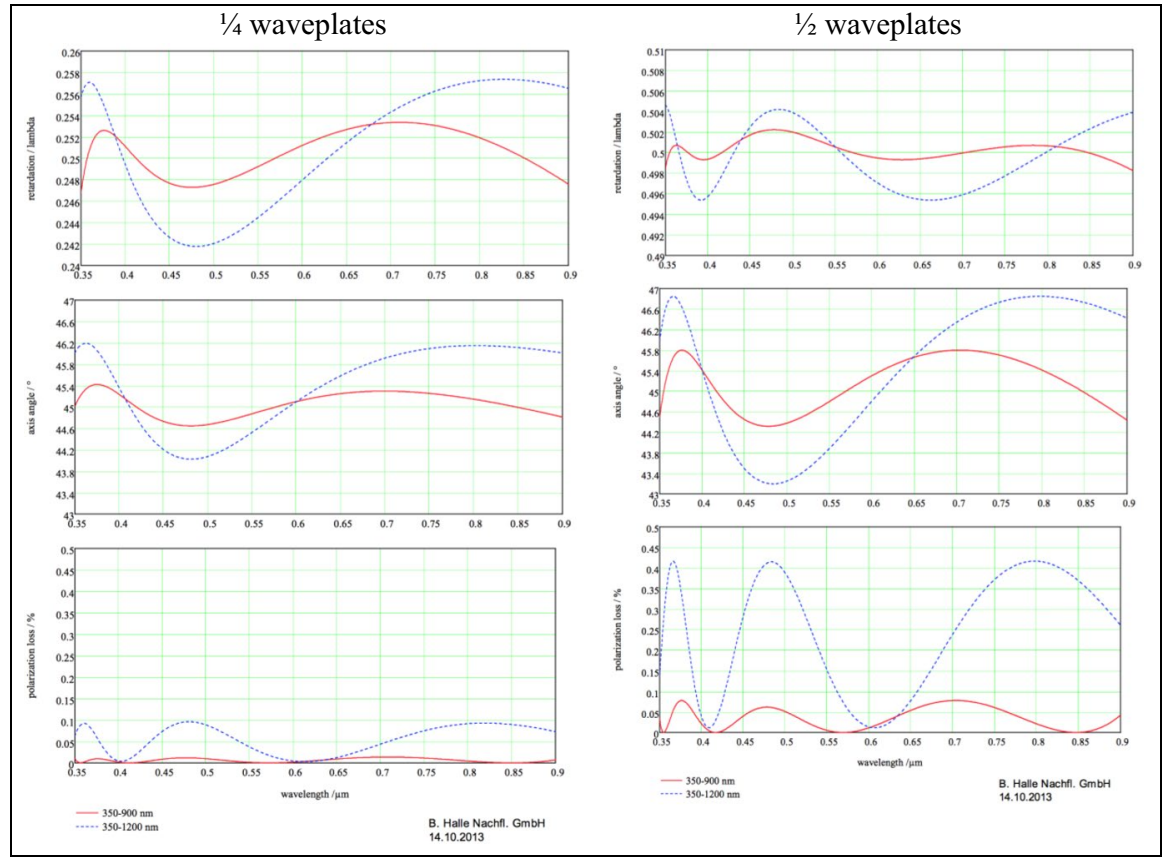

Fig. 5 Performance of both $1 / 2$ and $1 / 4$ waveplates

while the dashed blue curve is a standard product optimized for the $350-1200 \mathrm{~nm}$ passband.

\subsubsection{Polarizers}

For the polarizer component a single Wollaston prism is chosen with a $1^{\circ}$ beam deviation between the ordinary and extraordinary rays. This value of angular separation allows for a very long slit in the spectro-polarimetric observing mode.

The material selected was crystalline quartz over calcite and a single prism of $40 \times 40 \mathrm{~mm}$ cross section was selected. The deviation of $1^{\circ}$ is at a wavelength of $530 \mathrm{~nm}$. This means that in imaging mode the ordinary and extraordinary image of a given object can appear to be virtually separated by $5.2-5.7 \mathrm{~mm}$ apart at the slit plane (primary focal plane), depending on the wavelength [9]. Thus, for polarimetric imaging there will be a focal plane mask as shown in Fig. 6, to avoid the confusion and overlap between the ordinary and extraordinary images. In this figure we can see that there are 5 pairs of opening-obscuration aligned parallel to the beam separation of the Wollaston prism, covering the $8.3 \times 8.3$ arcmin FoV at the slit plane or primary focus. The sizes are given as both linear dimensions (mm) and angular dimensions (arcsec or arcmin).

The use of the venetian-blind mask means that to cover a given field, multiple images have to be taken. Since the obscured area is somewhat larger than the exposed area (due to the changing beam separation as a function of wavelength) 


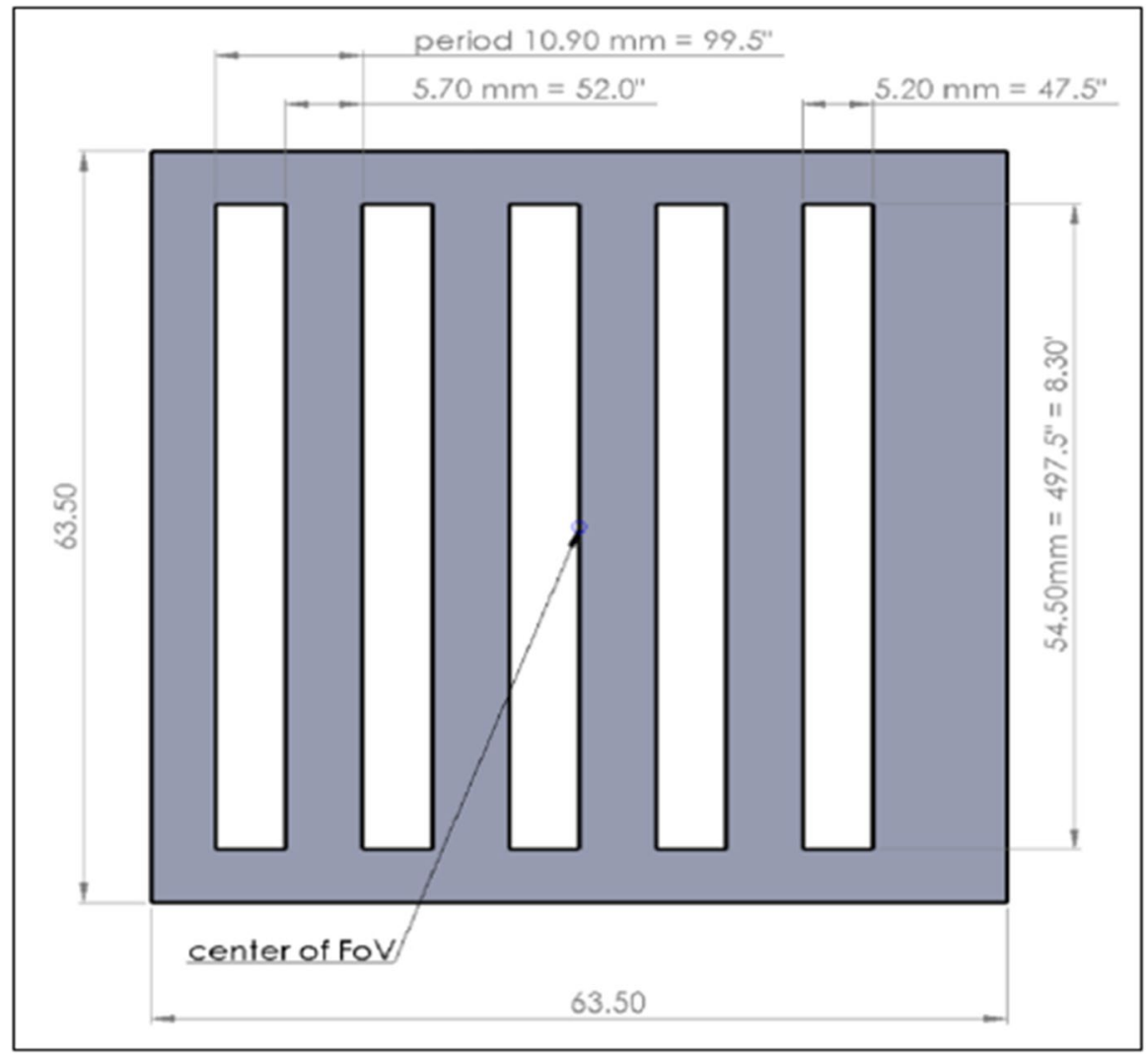

Fig. 6 Venetian blind focal plane mask for polarimetric imaging

two images taken with an angular shift of 52" of the telescope will cover $95.5 \%$ of the FoV. Thus, a third image is needed if the entire $100 \%$ of the FoV has to be recorded.

\subsection{Calibration unit}

The volume before the slit plane hosts the calibration light injection system. It is a $100 \mathrm{~mm}$ integrating sphere equipped with the required light sources (incandescent halogen bulb for continuum reference and flat fielding, and a ThAr arc lamp for spectrum calibration). The highly diverging beam exiting the sphere is refocused using a Fresnel lens to match the f/12.06 beam of the corrector. This unit can be inserted in place of the corrector, thus blocking the beam of the telescope and sending the calibration light towards the slit plane and the rest of the optical system as is shown in Fig. 7. 


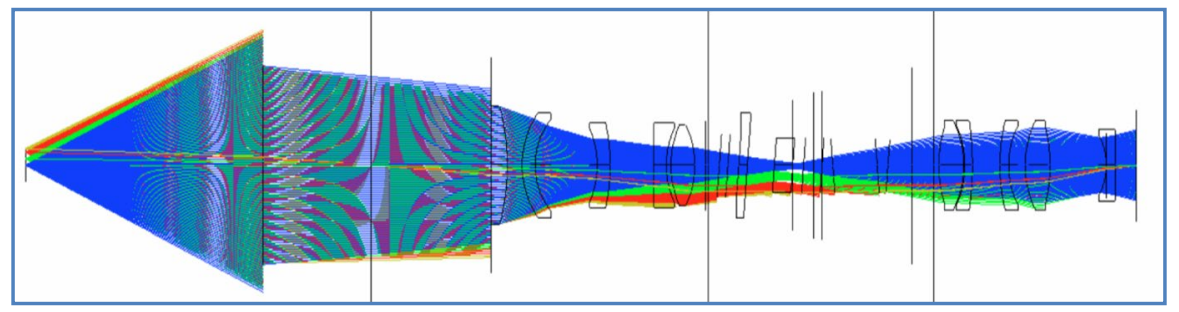

Fig. 7 Calibration system optical design

\subsection{Optical performance}

\subsubsection{Throughput}

All the optical materials were selected to allow for maximum possible transmission at $350 \mathrm{~nm}$ wavelength. Therefore, highly blue transmissive Ohara I-line glasses are used along with crystalline materials. All surfaces have a dielectric multi-layer anti-reflection coating, designed for 350-900 nm passband and the actual refractive index of the given lens elements. Although it requires individual coating runs for each of the glass types, maximum blue sensitivity was provided. The waveplates and Wollaston prism have a single MgF2 layer AR coating, and the cover plates of the retarders are fused silica. The total throughput of KFISP optical train (not including the telescope, filter transmission, CCD quantum efficiency or grating efficiencies) was estimated (Fig. 8). Also included in Fig. 8 the estimated total system, including surface AR coating.

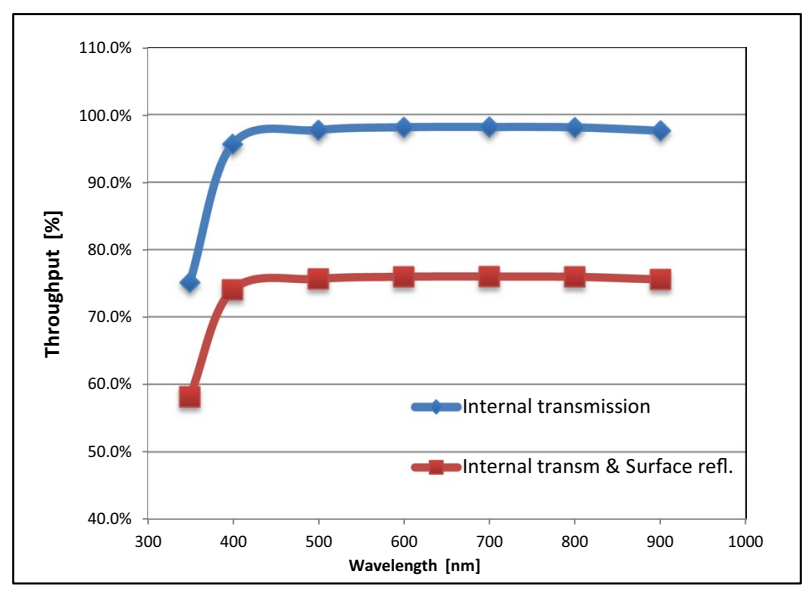

Fig. 8 Estimated throughput of the optical components 


\subsubsection{Imaging quality: imaging modes}

The image quality is shown for direct imaging in Fig. 9, using spot diagrams and encircled energy versus field position plots. As is shown in this figure, the box size is $54 \times 54$ microns or $4 \times 4$ pixels or $0.96 \times 0.96$ arc seconds. The design requirement was to achieve $80 \%$ Encircled Energy (EE) radius to be smaller than 7.5 microns. This requirement is met at four of the field positions shown in Fig. 9 for white light or photometric (without filter) imaging.

\section{Opto-mechanical design}

Figure 10 displays the overall instrument layout which identifies the individual components/subassemblies. There are three large circular plates which are separated by a series of double-layered carbon-fiber tubes. A rectangular instrument box is attached to the lower plate and it contains pull-out modules for the filter wheels, grisms and polarimeter components. The main science camera is attached to the bottom of the instrument box. The front end hosts the corrector, guider and slit assemblies, as well as the calibration system. It is connected to the main instrument hub via 6 bipods to provide a stiff, rigid structure. The instrument has nearly symmetrical mass distribution around the optical axis to equalize and minimize changes as a result of instrument rotation at certain telescope positions. The collimator lens assembly is protruding out of the middle hub towards the front end, supported by another 6

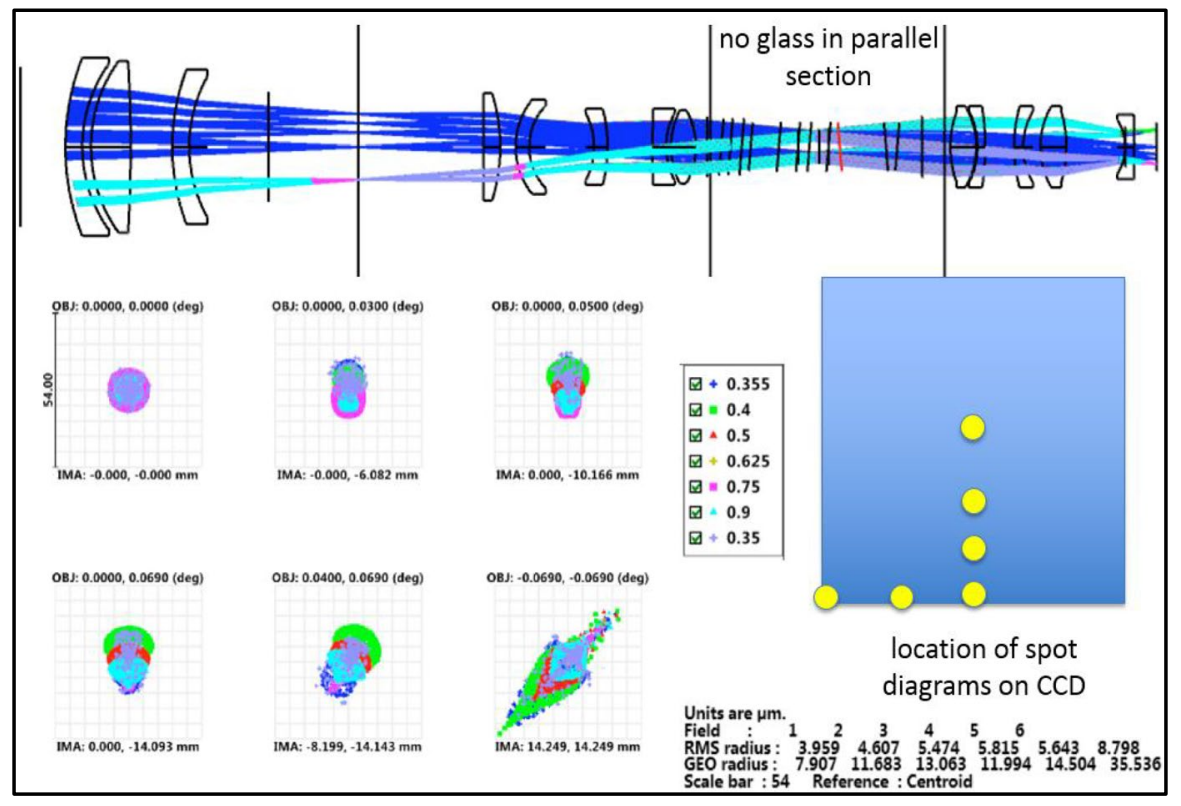

Fig. 9 Spot diagrams in white light or photometric (without filter) imaging 


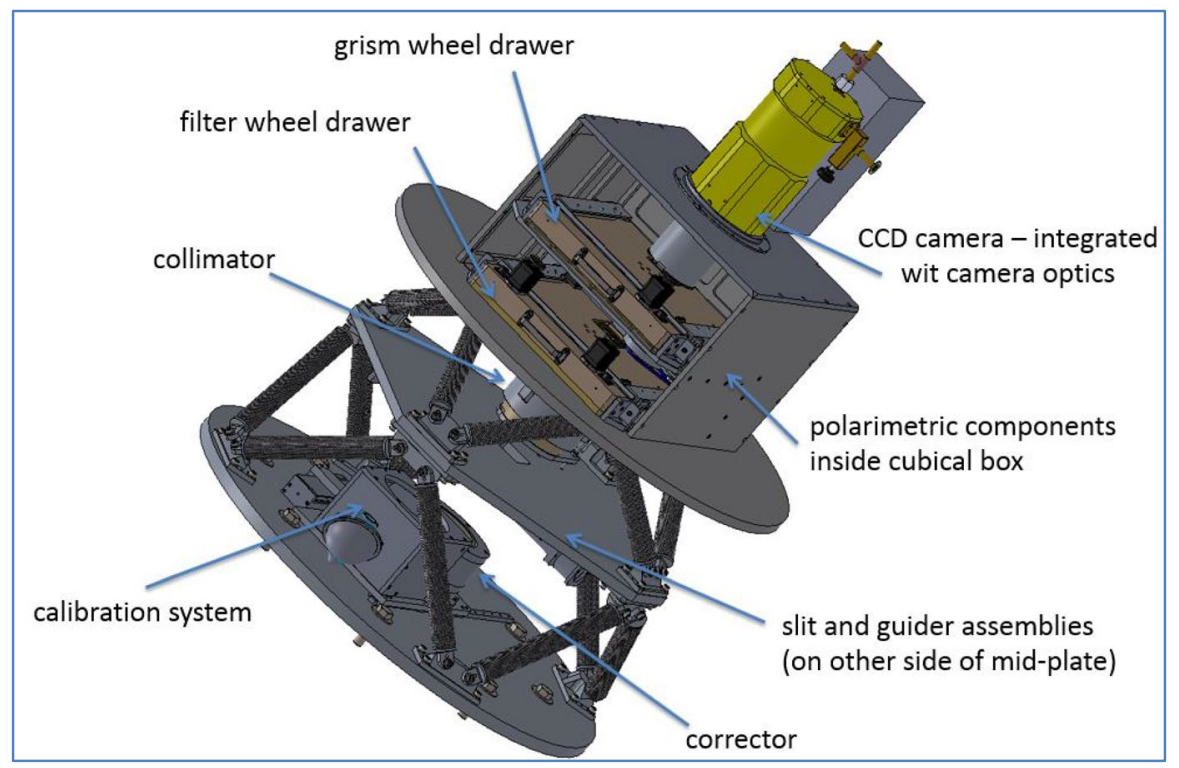

Fig. 10 Opto-mechanical design of KFISP

bipods to provide a stiff, rigid structure. The opto-mechanical parts of KFISP are fully enclosed with covers (shrouds) to avoid dust contamination.

The overall length of the KFISP, including the CCD shown, is $1343 \mathrm{~mm}$ and the mass of the instrument is about $275 \mathrm{~kg}$. The KFISP can be broken up into sections for assembly and maintenance.

Corrector lenses as well as all other optical elements are potted into metallic (aluminum) bezels using silicone RTV bond. The bond geometry is optimized for the given glass material and lens geometry, so the mounting is athermalized for the specified temperature range and optimized for lowest stress for $50{ }^{\circ} \mathrm{C}$ temperature change and minimal axial/radial displacement under gravity load.

\subsection{Calibration system and corrector assembly}

The calibration module has a linear stage driven by a stepper motor. The stage can be at one of two operational positions: observing, where the corrector lens in the beam and calibrating, when the calibration mirror/lamps are in the beam. This assembly is shown in Fig. 11. When the corrector is in the beam the starlight passes through the spectrograph. When the calibrator is in the beam the starlight is blocked. Now light from an integrating sphere is projected onto the calibrator mirror and injected into the spectrograph. The integrating sphere has two input ports and one output port. A thorium lamp filled with argon (Th-Ar) is used for spectral calibration. A quartz lamp is used for flat fields. 


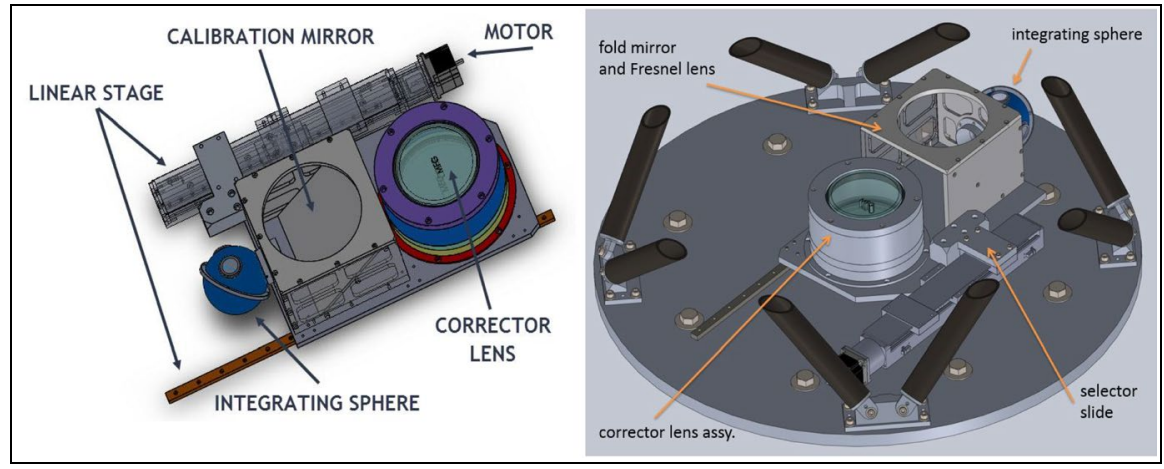

Fig. 11 Calibration system and corrector assembly

\subsection{Guider, slit selector subassembly and slit viewing camera}

Within the front end there are the guider, slit mask stage and slit viewer camera. A leadscrew drives the slit mask stage which contains an adjustable slit and three removable masks. There are four position sensors to indicate that the stage is at one of four standard positions: adjustable slit in the beam; imaging mask in the beam (empty mask for direct imaging); venetian blind mask in the beam (for polarimetry); pinhole mask in the beam (for engineering setup). These components are shown in Fig. 12. The spectroscopic mode of KFISP offers a 4' long slit, that is limited to $50^{\prime \prime}$ slit length in spectropolarimetric mode, due to the $1^{\circ}$ angular separation limit of the Wollaston prism. The adjustable slit is made from two silicon wafer mirrors that can be moved apart to create an infinitely variable slit width. Silicon wafers were chosen because they are readily available in very thin $(0.5 \mathrm{~mm})$ and flat (quarter-wave) die-cut sheets with very sharp edges (to create the slit) and can easily be aluminized. In practice the slit width can be changed from zero to about 5 arc seconds. A Vee-shaped decker plate hidden behind the slit adjusts the slit length.

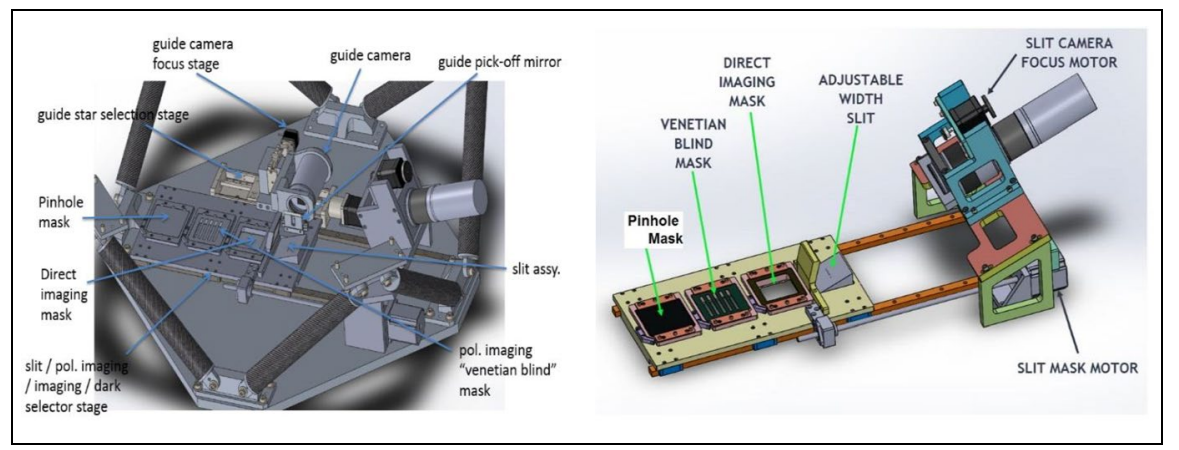

Fig. 12 Guider, slit selector assembly and slit viewing camera 
The pinhole mask contains a grid of $11 \times 11$ pinholes, each with $50 \mu \mathrm{m}$ circular opening and is used for focusing purposes of KFISP apart from the telescope.

The guider module allows a small pick-off mirror to move in the off-axis beam to direct light to a guide camera. Unlike the slit-viewing camera, which can only be used during spectroscopy, the guide camera can be used at all times in all observing modes. The guider camera is a QHY12 CCD with $24.0 \times 16.4 \mathrm{~mm}\left(3.6^{\prime} \times 2.5^{\prime} \mathrm{FoV}\right)$. The camera has $4610 \times 3080$ pixels with a pixel size of $5.12 \mu \mathrm{m}$. These dimensions give $0.046^{\prime \prime} /$ pixel sampling or $0.19^{\prime \prime} /$ pixel sampling when binned to $4 \times 4$, which can sweep a $3.6^{\prime} \times 12^{\prime}$ field of view by moving it along a single axis.

The slit viewing camera can only be used when the adjustable slit is in the beam. A small mirror, mounted to the moveable stage, reflects light off the adjustable slit to a camera. The camera is not mounted on the stage so it can only see the adjustable slit when the slit is in the beam. The camera (QHY CCD) is attached to the rear of a Canon $100 \mathrm{~mm}$ lens. It images at the slit so that stars can be placed down the slit. A stepper motor drives a belt to turn the focus ring of the camera lens. Achieving correct focus of the KFISP is a multi-step process. First, stars must be in focus on the slit pate. The slit-viewing camera is focused on the slit and then the telescope secondary is moved until stars are in focus in the slit-viewing camera. Finally, the collimator of the KFISP is moved to achieve sharp images at the detector. Focus can be maintained by monitoring the slit-viewing or guider camera. In practice the telescope focus changes more than the internal focus of the KFISP.

\subsection{Collimator focus module}

The collimator is a custom lens that serves two purposes. It creates a parallel beam for the filters, spectroscopic and polarimeter elements, and it also used to focus the KFISP as the subsequent camera lens is fixed. The collimator uses an absolute encoder with a spring-loaded plunger. It never loses position and is not affected by any leadscrew errors or backlash. The collimator module in cross-sectional view is as shown in Fig. 13. The collimator is captive inside the KFISP assembly and cannot be removed without separating the upper truss assembly from the central plate by removing the truss screw. The collimator cross-sectional view reveals the inner workings of the module. A series of 5 custom lenses are used in the optical design. They are held in the tube by a series of precision spacers. The entire inner tube is driven by a leadscrew. The total movement is very small, about $10 \mathrm{~mm}$. The inner tube rides on three pairs of ball bearing races in a Crayford design.

\subsection{Filter and grism wheel modules}

Filter and grism wheel modules are mounted in removable drawers as previously shown in Fig. 10. This allows filters to be inserted or removed and for general servicing. Each wheel rides on a pair of deep grove radial bearings as shown in Fig. 14. There is a pattern engraved into the rim of each wheel. A spring-loaded detent arm holds the wheel in place and there is no power on the motor when it is not moving. A series of roller switches rides in one of the patterns and provides 


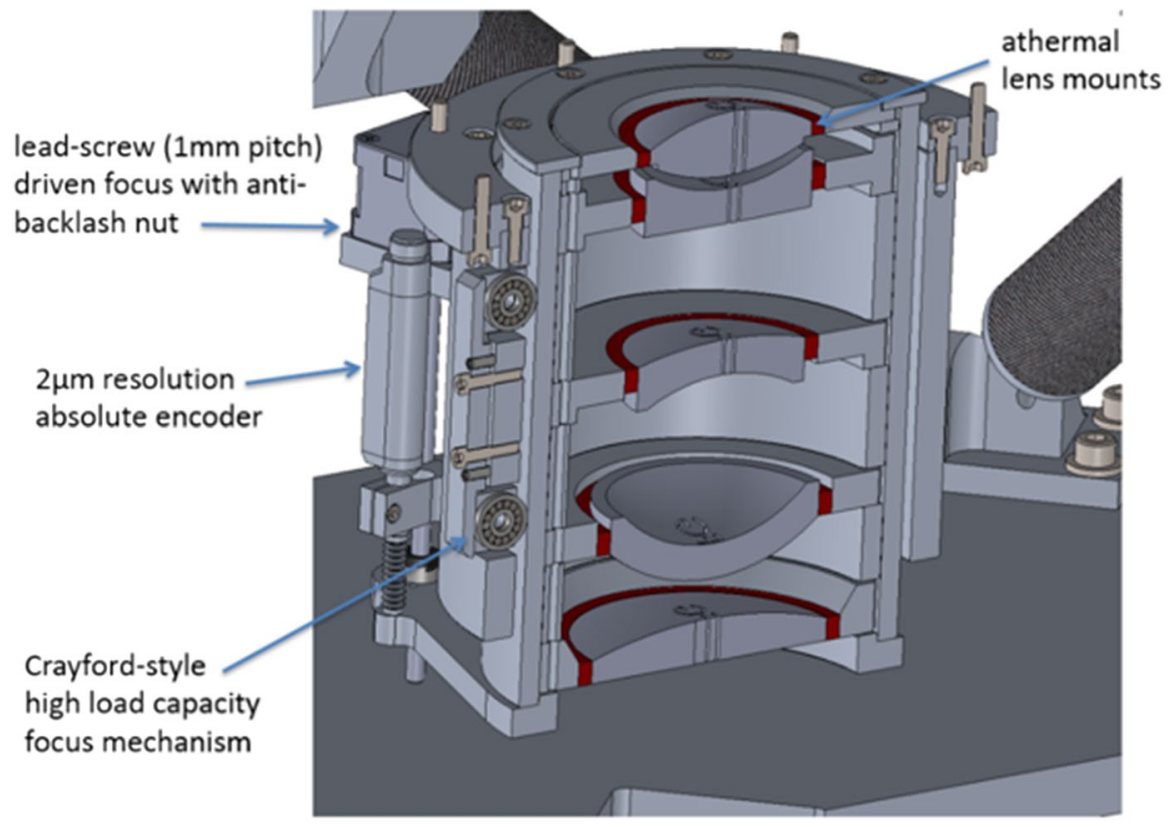

Fig. 13 Collimator cross sectional view

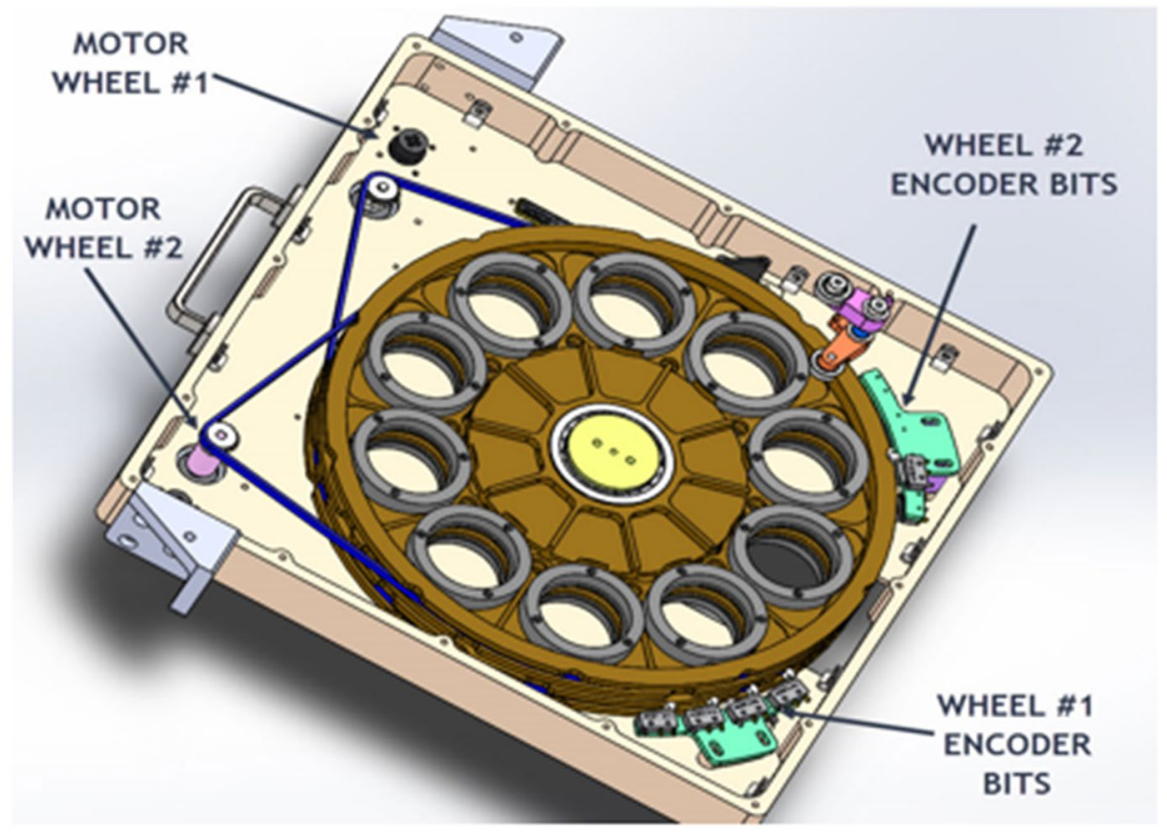

Fig. 14 Dual filter wheel module 
absolute binary encoding of the wheel. Each wheel has 10 openings permitting the use of 18 round filters and leaving a single opening in each wheel for white light imaging.

The grism wheel operates in an identical manner to the filter wheel except there is only one wheel with five positions. They are labelled 0 through 4 and the gratings are also identified. Similar to filter wheels, grism wheel employs preloaded mechanical detents to achieve high repeatability in position.

\subsection{Polarimeter module}

The polarimeter module is shown in Fig. 15. The module allows for independent insertion and rotation of both $1 / 4$ and $1 / 2$ waveplates. A Wollaston prism is also independently moved into the beam. A venetian blind is available on the slit mask stage for use with polarimeter observations. The waveplates can be rotated with $0.5^{\prime}$ precision.

\subsection{Camera lens/CCD detector module}

The detector is an Astronomical Research Camera [10] product using a thinned, back-illuminated E2V sensor with $2048 \times 2048$ pixels, each 13.5 microns square. It is cooled using liquid Nitrogen to $-110^{\circ} \mathrm{C}$. The standard dewar window was replaced with a lens which is the last element in the camera lens as is shown in Fig. 16. The lens barrel can be removed from the dewar faceplate window for servicing the shutter. The camera can be operated in single readout mode from either the left or the right side, or in dual readout mode from both sides simultaneously [11].

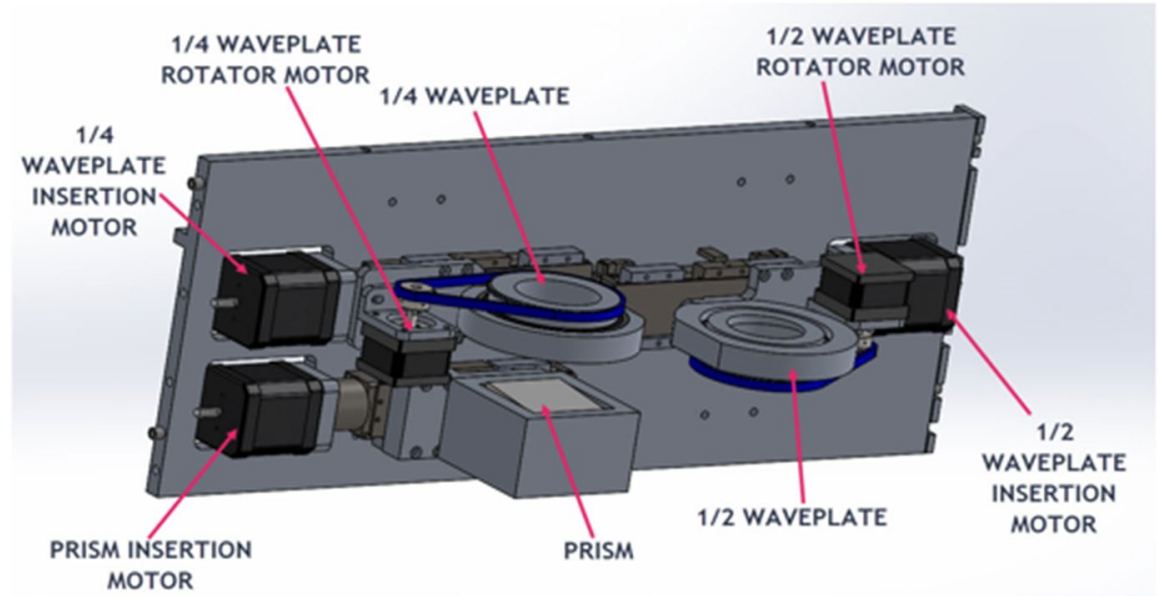

Fig. 15 Polarimeter module of KFISP 


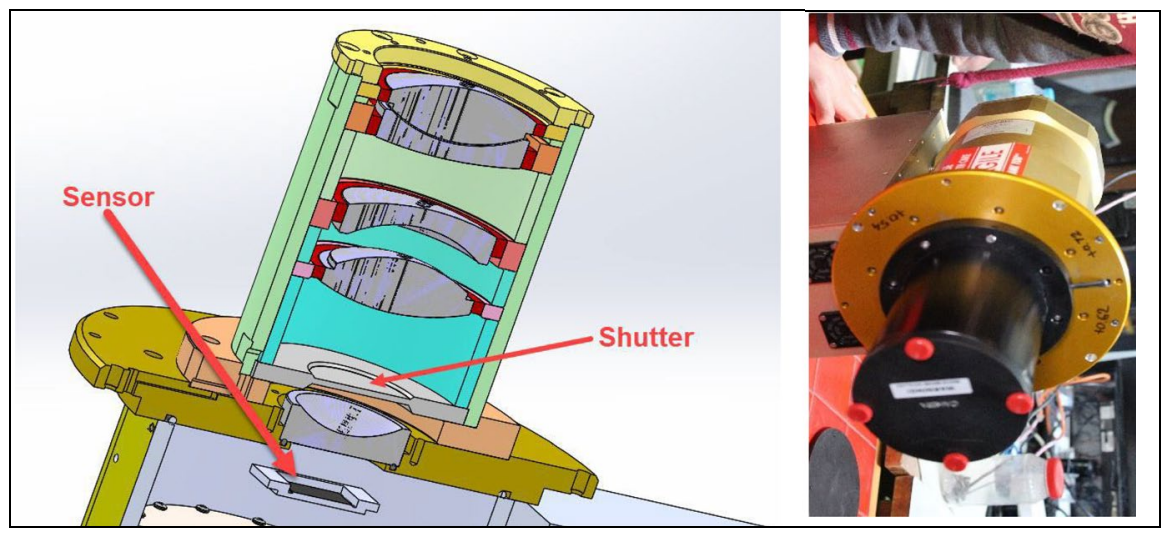

Fig. 16 Camera lens/CCD detector module

\section{KFISP control software}

\subsection{Motion control summary}

The KFISP uses a National Instruments CompactRio 9032 for command and control [12]. There are 16 axes of motion in the KFISP. They are managed by four all motion EZ-4AXIS controllers, each of them driving four stepper motors. They talk to the Compact Rio using a RS-432 communication bus. There are 32 digital inputs controlling various components of the previously mentioned modules. The CompactRio 9032 device communicates with the KFISP control computer (located in the warm room) over a wireless network.

In addition, there is an Icron Ranger 2324 USB 2.0 fiber-optic extender that goes from the KFISP to the guider computer that controls the guider and slit viewing cameras through MaxIm DL software. A separate fiber optic cable is also used for the ARC science camera that goes to the PCI-based card in the KFISP computer [11].

\subsection{KFISP control program}

The KFISP control program is a GUI high-level software interface for the control of the KFISP axes. The main GUI presents a single window which controls all of the axes as shown in Fig. 17. This window is divided into small sections for each module of KFISP.

At the start of software, the KFISP initializes the axes and place the stages in known positions. At the top of the window, a large button labeled with a red stop sign and the words "Stop all" can be used to stop all motions in the spectrograph. At the bottom right corner, a button labeled "Console" can be used to switch to a console view. The console view can be used to view raw commands being sent to the CompactRio and to send custom commands [11]. 


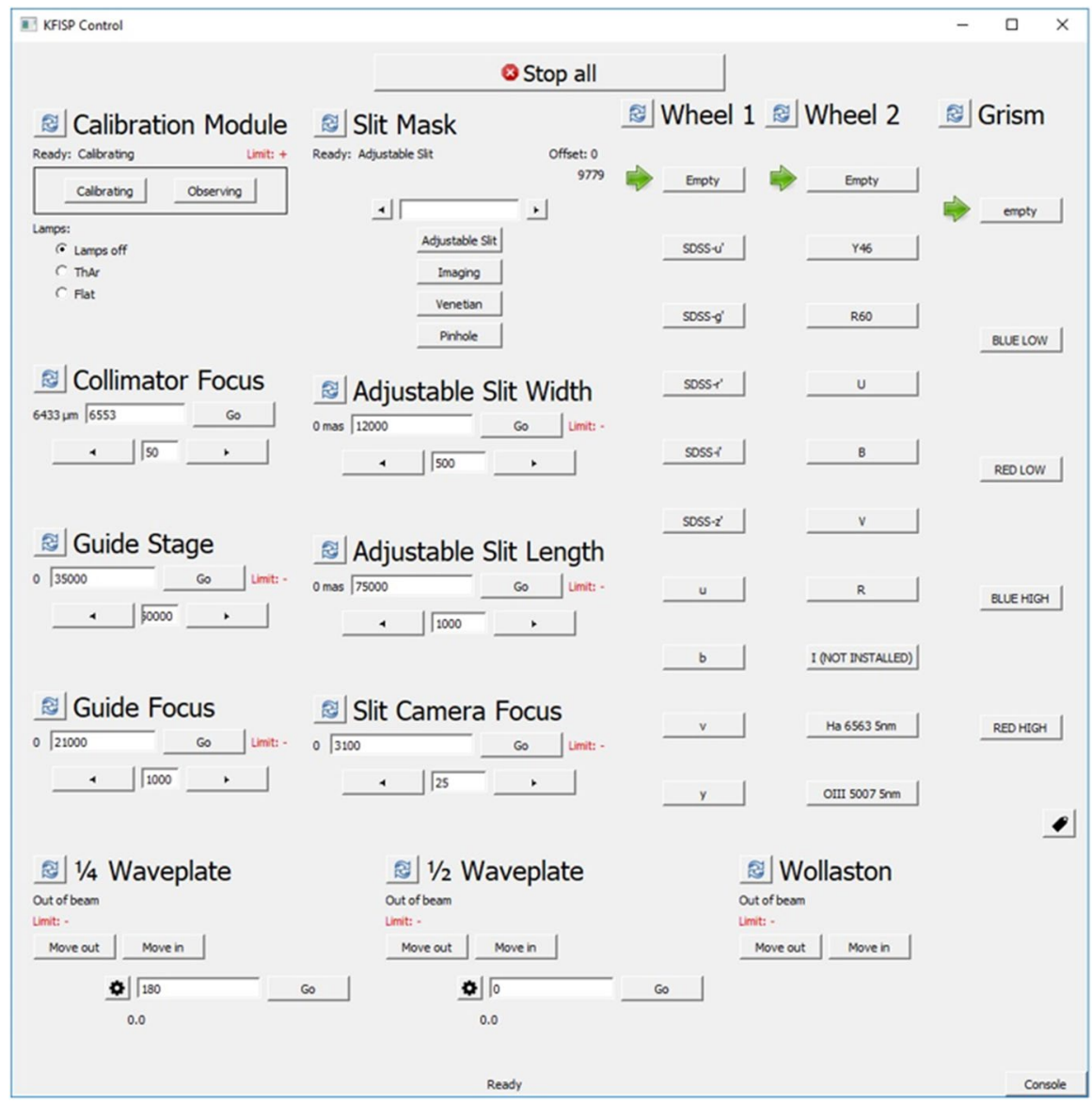

Fig. 17 The KFISP Control GUI

For each control axis on KFISP, the GUI provides a button showing two arrows forming a circle. These buttons are used to re-initialize the specified axis. The initialization procedure differs from axis to axis, and the button may have no effect if an axis is already in its initialized state. Most axes are initialized to their negative limits. The waveplate rotation axes have a home sensor that defines the initialization state. The filter wheels and grism wheel are considered initialized at any valid filter or grism position.

\subsection{ARC camera main control interface}

The main interface controlling the CCD camera is shown in the left part of Fig. 18. As is shown, it is divided into an upper and a lower section. The upper section is used to collect information about how the camera should take an exposure. The first two fields: observer(s), and notes, are stored into the image header. The exposure 


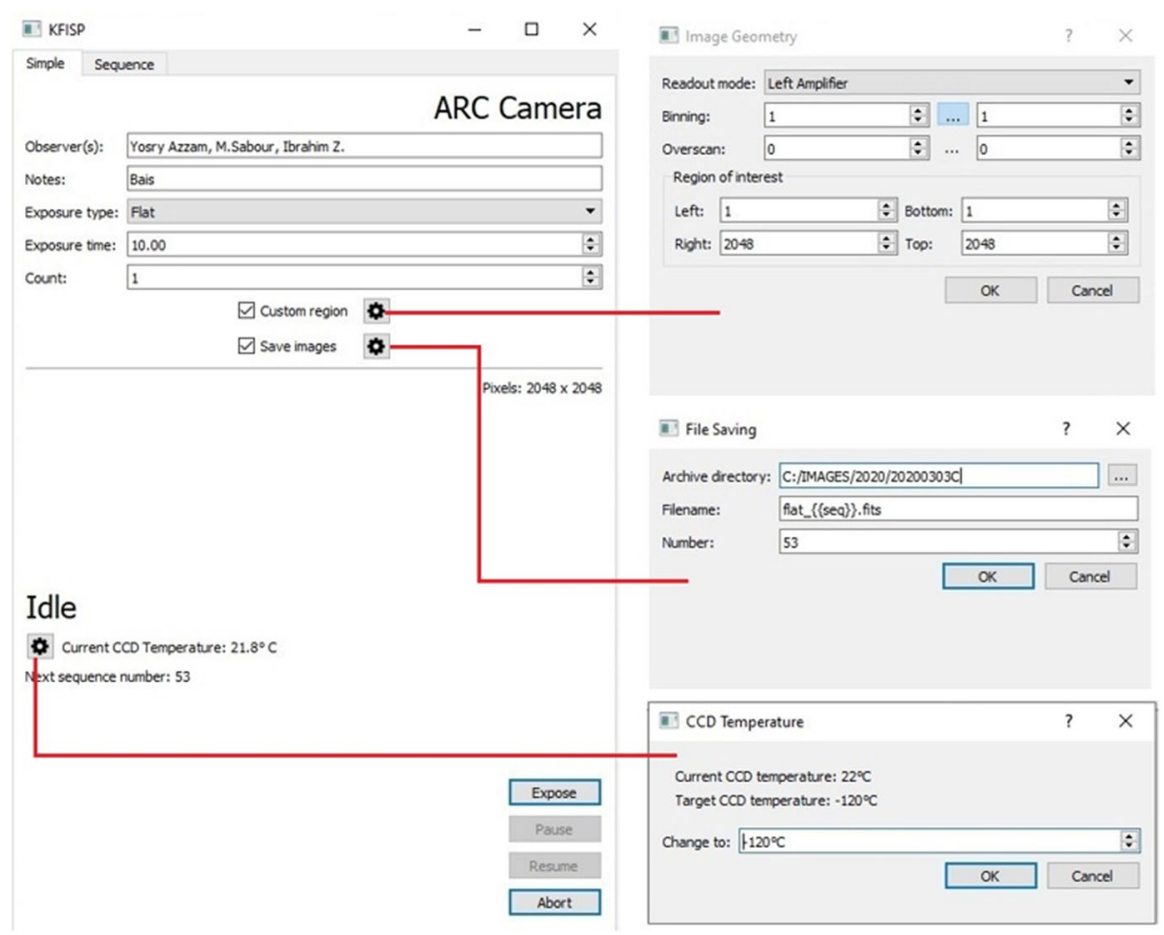

Fig. 18 The main camera control window: Simple Tab and related popup windows

type is recorded into the FITS header, and also selects the behavior of the shutter. The exposure types available are light, bias, dark, flat and sky flat. The main camera control window has two tabs namely simple and sequence. The simple tab is used to acquire one or more frames with the same parameters and filters whereas the sequence tab is used to perform photometric sequences.

\section{Sample of first light observations}

Samples of first-light observations obtained from KFISP during its test phase are shown in Figs. 19, 20, 22 and 25, which show various images for different observing modes. These images are introduced to show that the function of each observation mode is clear. Figure 19 displays the M67 open star cluster in direct imaging mode in V-band filter with an exposure time of $15 \mathrm{~s}$. Simple analysis of the data in this image reveals that the mean FWHM $=1.3 \pm 0.1$ arcseconds and the median $\mathrm{FWHM}=1.2 \pm 0.1$ arcseconds. For the stars with relative error $<10 \%$ (i.e. e_Vmag/ Vmag $<0.1$ ), the limiting magnitude of the faint stars is 20.6 mag.

Figure 20 displays the continuum level normalized spectrum for omicron And (HD217675) star using Red M Grism. The X-axis is the wavelength in Angstrom while the Y-axis is the relative intensity. 


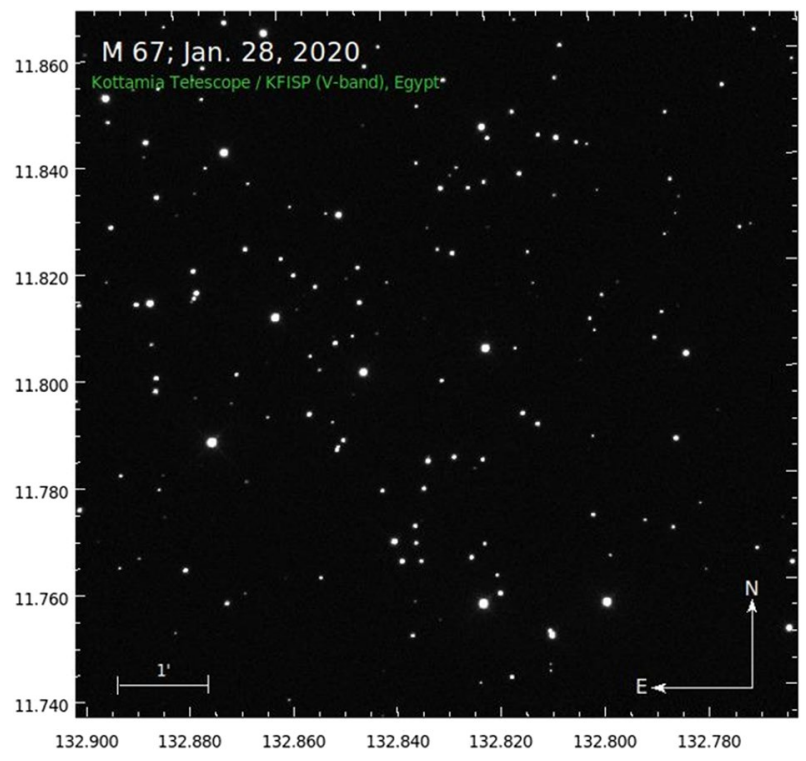

Fig. 19 Sample of first light observations for direct imaging mode

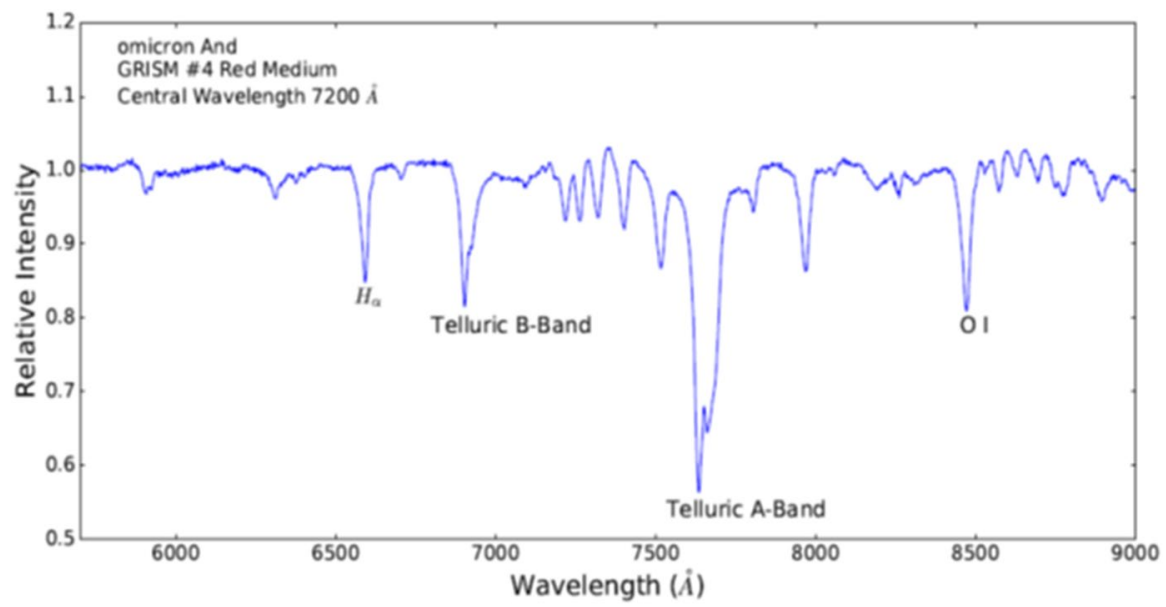

Fig. 20 Spectroscopic wavelength calibrated spectrum of first light observations of omicron And (HD217675) using Red M Grism

In the imaging polarimery observing mode, the instrumental polarization was measured from the unpolarized star HD103095 [13] which is displayed in Fig. 21. As is shown, instrumental polarization is small in $\mathrm{B}, \mathrm{V}$, and $\mathrm{R}$ bands $(<0.05 \%)$. At U-band, it is slightly larger than other bands but still small enough $(\lesssim 0.1 \%)$.

Figure 22 shows an image of strongly polarized star HD154445 by imaging polarimetry mode [14]. The image indicates that five pairs of truncated 


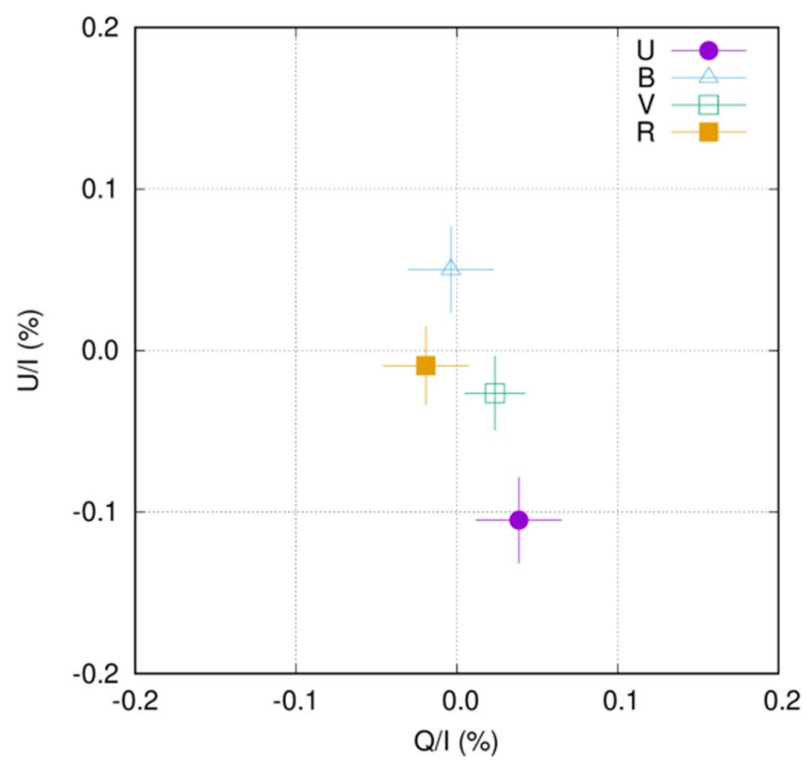

Fig. 21 Instrumental polarization of KFISP measured from unpolarized star HD103095

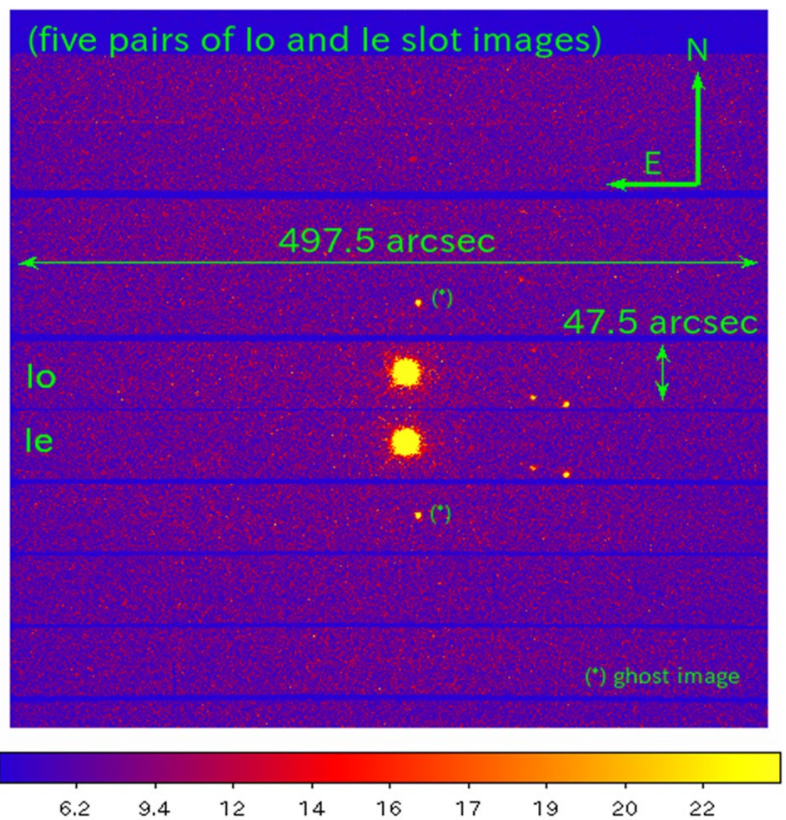

Fig. 22 Polarimetric mode of first light observations of strongly polarized standard star HD 154445 
rectangular sky fields of ordinary and extra-ordinary rays (o-ray and e-ray) split by the Wollaston prism are exposed on the detector. A simple analysis of the data in this image is implemented by calculating calibration values of the instrument using images of this object and its literature values [14, 15]. After that, another standard star object (HD161056) is observed and images of this object are reduced as if it is not a standard star but as general target star, using calibration values deduced above from HD154445. The results obtained indicating the polarization degrees and instrumental position angles at B, V, and R-band are as shown in Figs. 23 and 24. As is shown, the observed values of $P(\lambda)$ and $P A(\lambda)$ are roughly consistent with literature values, although the values at R-band seem to differ slightly, which may be a consequence of insufficient calibration or for other reasons because enough observational testing has not yet been done.

Figure 25 shows an image of the unpolarized star HD103095 which was taken using spectro-polarimetric mode. Stellar image on the slit aperture is dispersed by inserted "Blue L" grism and split into o-and e- rays by the Wollaston prism. Eventually, we obtain a pair of perpendicularly polarized spectra of the target object. Analysis of the data in this image is still needs to be implemented to assess the performance of the spectro-polarimetric mode which is expected to be done in future work for a comprehensive assessment of the instrument in all of its working modes.

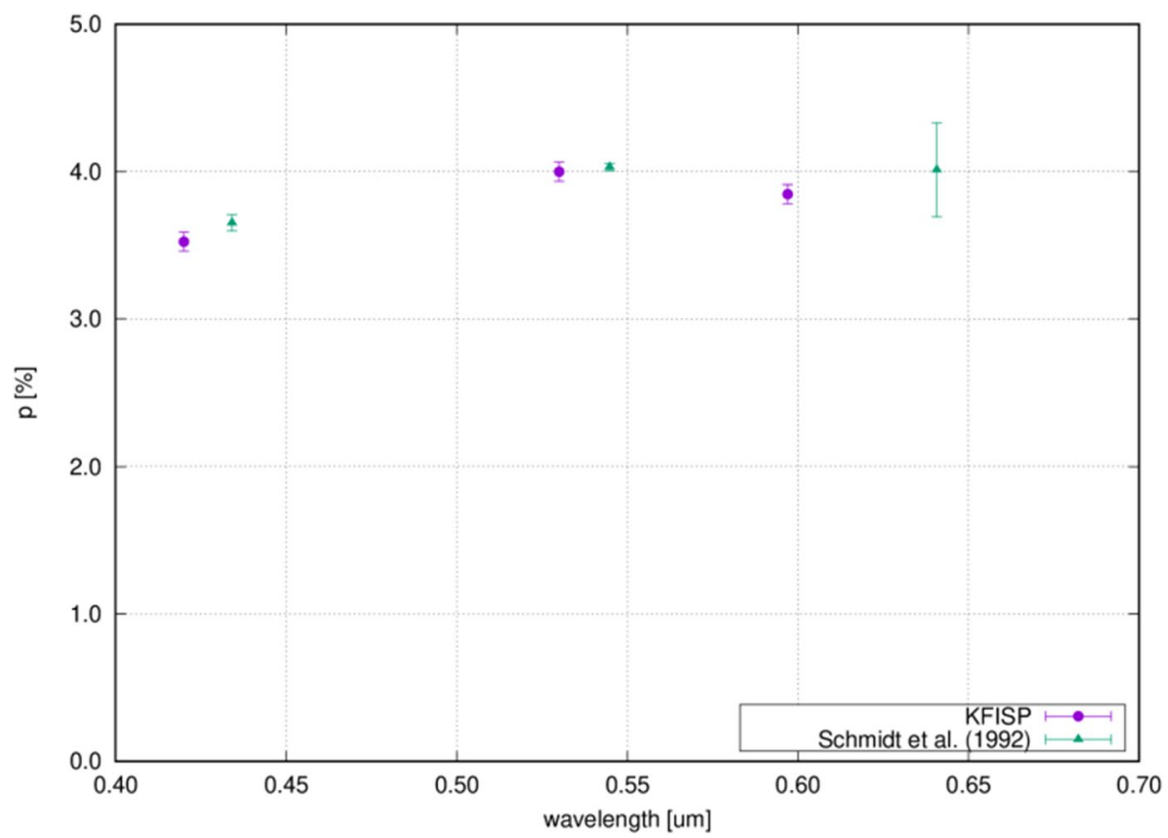

Fig. 23 Polarization degree of HD161056 compared with literature values 


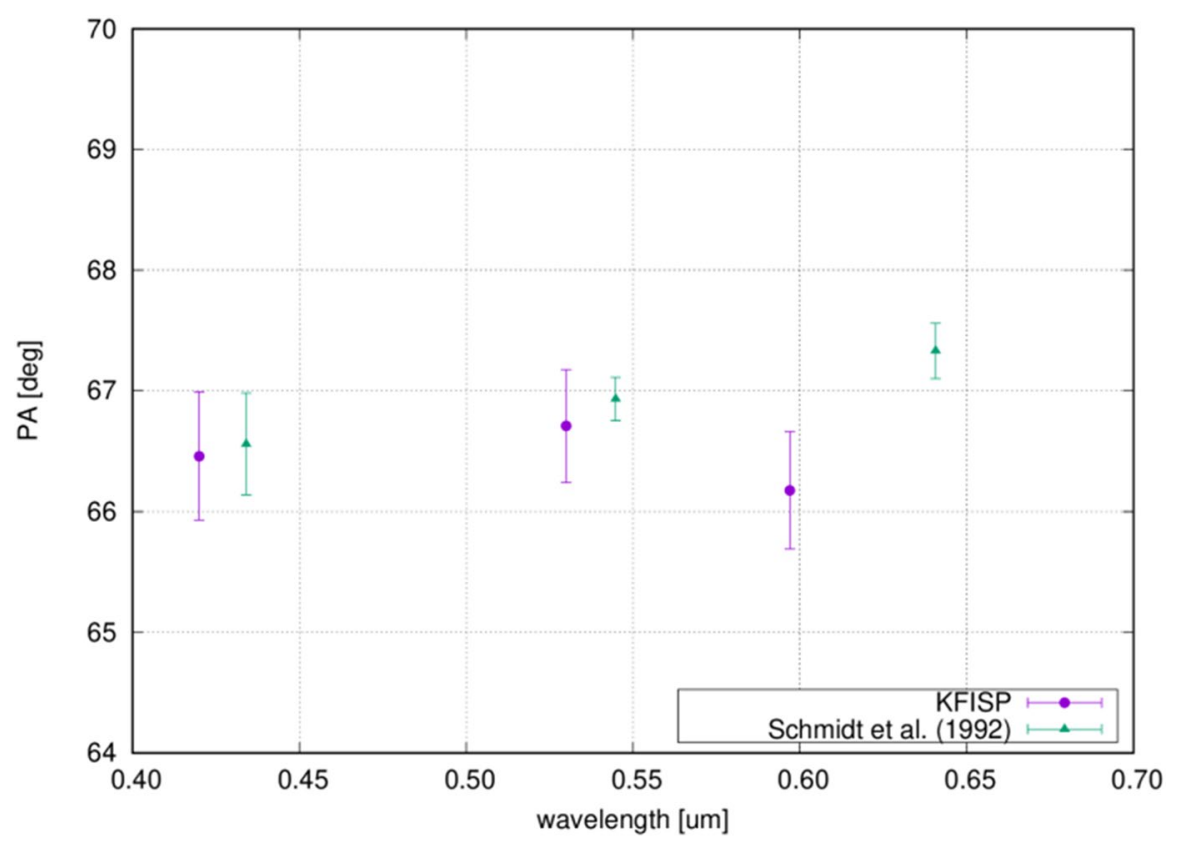

Fig. 24 Position angles of HD161056 compared with literature values

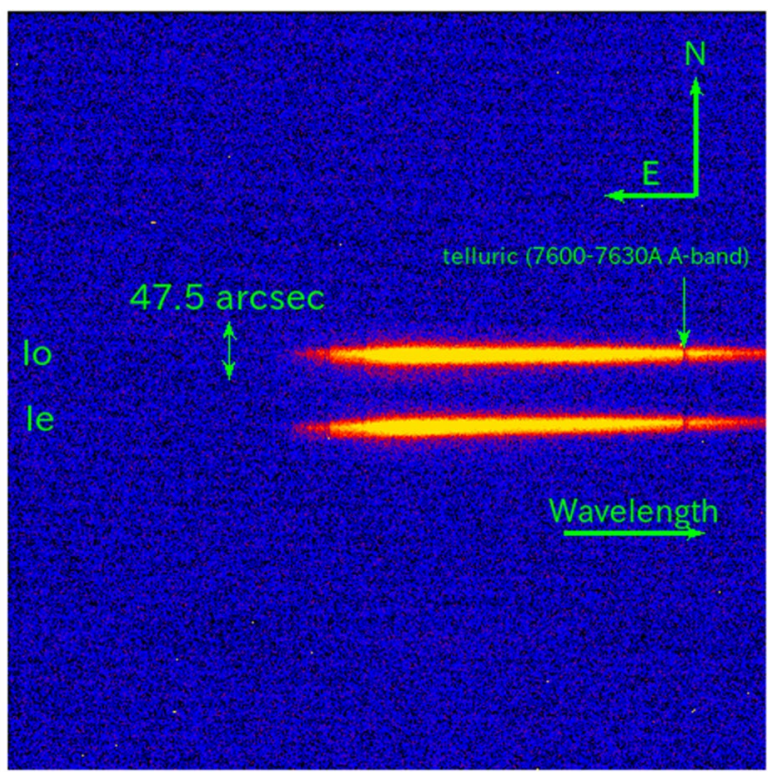

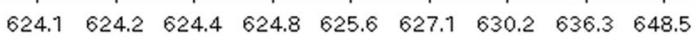

Fig. 25 Spectro-polarimetric mode of first light observations of unpolarized star HD103095 


\section{Conclusions}

The Kottamia Faint Imaging Spectro-Polarimeter (KFISP) has been introduced in this paper including its optical and opto-mechanical design. In addition, the KFISP control system and software GUI interface have also introduced. In this framework, various components describing the KFISP have been explained in modular form to demonstrate the functionality of the complete instrument which gives an effective focal ratio of F/6.14. This design increased the FoV of the telescope at its Cassegrain focus from 2.8 arcminutes to 8.2 arcminutes for its direct imaging mode. In addition, it added new modes of operation that were not available at the Kottamia telescope. These are: spectroscopic, polarimetric imaging, and spectro-polarimetric. We have also introduced the optical performance of the instrument as well as its imaging quality. The KFISP was planned and designed to perform observational research based on extended observations of transient objects like GRBs, supernovae and counterpart of the gravitational wave events, and we aimed to design a versatile, multi-mode optical instrument to record various information of the objects without any additional work.

Although COVID-19 corona virus pandemic caused a delay and retardation of the full usage and getting large-scale observations from the device, we introduced a sample of the first light observations obtained from the instrument in different observing modes. The observations prove that the instrument is behaving well and will soon be used extensively to demonstrate its image quality and obtain observations which will be analyzed and published in the astronomical society journals.

Acknowledgements The authors would like to thank all colleagues who took part in the design, installation, and attending the tests made to KFISP. In particular, we thank our colleagues who rested in peace before seeing this instrument working. Those are: Prof. M. Hamdy, Prof. I. A. Issa, and Dr. Gamal B. Ali. Many thanks are due to Prof. A. M. I. Osman, Prof. M. S. Abo-Elala, and Prof. A. A. Haroon. We also thank Kottamia engineering staff: Mohamed Ismail, Islam Helmy, Doaa Elsayed and Mohamed Abdelkarim for their help and support they gave to KFISP installation and test. Finally, we thank astronomers M. S. Darwish, Ahmed M. Fouad, Ola Ali and Mona Molham for attending the installation and test of KFISP. This research makes use of the data available by both KFISP spectrograph funded by the Egyptian science and technology development fund STDF No. 2531 and the attached $2 \mathrm{k} \times 2 \mathrm{k}$ CCD camera funded by the STDF No. 5217. The authors are thankful to STDF for funding both projects and would like to acknowledge the PI's of both projects.

\section{References}

1. Azzam Y.A., Ali G.B., Elnagahy F., Ismail H.A., Haroon A., Selim I.: Current and future capabilities of the 74-inch telescope of kottamia astronomical observatory in Egypt. In: Haubold H., Mathai A. (eds.) Proceedings of the Third UN/ESA/NASA Workshop on the International Heliophysical Year 2007 and Basic Space Science. Astrophysics and Space Science Proceedings. Springer, Berlin

2. Hassan, S.M.: Upgrading the 1.9-m Kottamia telescope. Afr Skies 2, 16-17 (1998)

3. Issa, I.A., Gamal El Din, A.I.: Astronomical research activities with the 74 inch telescope at Kottamia observatory. Astrophys. Space Sci. 118, 87-93 (1986)

4. Hassan, S.M.: Kottamia telescope upgrading- from developing basic space science worldwide. A Decade of UN/ESA workshops. In: Wamsteker, W., Albrecht, R. and Haubold, H. (eds.), pp. 237240. Springer, Netherlands (2004) 
5. Azzam, Y.A. et al.: (KFISP) Kottamia faint imaging spectro-polarimeter: opto-mechanical design and performance analysis. In: Proc. SPIE 11447, Ground-based and Airborne Instrumentation for Astronomy VIII, 114479U (2020). https://doi.org/10.1117/12.2561664

6. Kawabata, K.S., Nagae, O., Chiyonobu, S., Tanaka, H., Nakaya, H., et al.: In: Proc. SPIE, vol. 7014, p. 70144 (2008). https://doi.org/10.1117/12.788569

7. Kashikawa, N., Aoki, K., Asai, R., Ebizuka, N., Inata, M., et al.: Publications of the Astronomical Society of Japan, vol. 54, pp. 819-832. (2002). https://doi.org/10.1093/pasj/54.6.819

8. Pancharatnam, S.: In: Proc. Indian Acad. Sci., vol. A41, p. 137 (1955)

9. Kawabata, K.S., Ebizuka, N., Sasaki, T., Sekiguchi, K., Iye, M., Aoki, K., Yoshida, M, et al.: Properties of FOCAS optical components. In: Proc. SPIE 4841, Instrument Design and Performance for Optical/Infrared Ground-based Telescopes (2003). https://doi.org/10.1117/12.461923

10. Astronomical Research Cameras (ARC), Inc. http://www.astro-cam.com. Accessed 6 Nov 2020

11. Azzam, Y.A., Elnagahy, F.I.Y., Zead, I., Brondel, B., Mack, P.: (KFISP) Kottamia Faint Imaging Spectro-Polarimeter: software, motion control, performance and results. In: Proc. SPIE 11447, Ground-based and Airborne Instrumentation for Astronomy VIII, 114479W (2020). https://doi.org/ $10.1117 / 12.2561665$

12. CompactRIO Systems. https://www.ni.com/en-lb/shop/compactrio.html. Accessed 5 Nov 2020

13. Serkowski, K.: Planets, stars, and nebulae: studied with photopolarimetry. In: Proc. Of IAU Colloq. 23, Gehrels, T. (ed.). University of Arizona Press, p. 135 (1974)

14. Schmidt, G.D., Elston, R., Lupie, O.L.: The Hubble Space Telescope northern-hemisphere grid of stellar polarimetric standards. Astron. J. 104, 1563 (1992). https://doi.org/10.1086/11634

15. Wolff, M.J., Nordsieck, K.H., Nook, M.A.: A medium-resolution search for polarmetric structure: moderate Y reddening sightlines. Astron. J. 111, 856-864 (1996)

Publisher's Note Springer Nature remains neutral with regard to jurisdictional claims in published maps and institutional affiliations.

\section{Authors and Affiliations}

\section{Yosry A. Azzam ${ }^{1}$ D . F. I. Y. Elnagahy ${ }^{1,2} \cdot$ Gamal B. Ali $^{1}$ - A. Essam ${ }^{1}$. Somaya Saad ${ }^{1,2} \cdot$ Hamed Ismail $^{1} \cdot$ I. Zead ${ }^{1,2} \cdot$ Nasser M. Ahmed $^{1}$. Michitoshi Yoshida ${ }^{3} \cdot$ Koji S. Kawabata ${ }^{4}$ Hiroshi Akitaya ${ }^{4,5} \cdot$ A. Shokry ${ }^{1}$. Y. H. M. Hendy ${ }^{1} \cdot$ Ali Takey $^{1}$ - G. M. Hamed ${ }^{1,6} \cdot$ Peter Mack $^{7}$}

Yosry A. Azzam

y.azzam@nriag.sci.eg

1 National Research Institute of Astronomy and Geophysics (NRIAG), Helwan, Cairo 1142, Egypt

2 Kottamia Center of Scientific Excellence for Astronomy and Space Science KCScE, Cairo, Egypt

3 National Astronomical Observatory of Japan, Subaru Telescope, 650 North A'ohoku Place, Hilo, HI 96720, USA

4 Hiroshima Astrophysical Science Center, Hiroshima University, 1-3-1 Kagamiyama, Higashi-Hiroshima, Hiroshima 739-8526, Japan

5 Planetary Exploration Research Center, Chiba Institute of Technology, 2-17-1 Tsudanuma, Narashino, Chiba 275-0016, Japan

6 Department of Astronomy and Space Sciences, Istanbul University, Beyazit, 34119 Istanbul, Turkey

7 Astronomical Consultants and Equipment, Inc., Tucson, AZ, USA 